\title{
DISTRIBUTION OF THE SUM OF RANDOMLY PHASED COMPONENTS
}

\author{
BY \\ W. R. BENNETT \\ Bell Telephone Laboratories, Murray Hill, N. J.
}

The so-called problem of random flights has engaged the attention of many investigators. In the general form we are required to find the distribution of the sum of $n$ vectors with specified lengths and randomly distributed directional angles. From the standard application of complex algebra to sinusoidal waves, it is clear that the two-dimensional form of the problem, with which we shall be concerned here, applies directly to the distribution of the sum of $n$ sine waves with equal frequencies, specified amplitudes, and random phase angles. By a simple extension which is reproduced in the appendix, we may also show equivalence with the distribution of the envelope of $n$ sine waves of incommensurable frequencies when all frequencies present are high relative to the difference between the lowest and highest. Applications thus exist to such diverse problems as random walks, in terms of which Pearson originally phrased the problem in $1905,^{1}$ and multichannel carrier telephony, which happens to be the stimulating influence behind the present investigation.

A concise summary of the general problem and its solution has been given by Watson. ${ }^{2}$ From these results, we take Kluyver's formula for the distribution, namely

$$
P_{n}(r)=r \int_{0}^{\infty} J_{1}(r t) d t \prod_{m=1}^{n} J_{0}\left(a_{m} t\right)
$$

where $P_{n}(r)$ is the probability that the sum of $n$ components having individual magnitudes $a_{1}, a_{2} \cdots a_{n}$ is less than $r$. We point out that the special case of $n=1$ is trivial, the case of $n=2$ is resolvable in terms of elementary functions, and the case of $n=3$ can be evaluated by elliptic integrals, ${ }^{3}$ so that the infinite integral presents no real computing problem for $n$ less than four. For large values of $n$, the integral may be calculated satisfactorily from asymptotic series in the manner indicated by Rayleigh. ${ }^{4}$ There remains considerable difficulty, however, in obtaining accurate numerical results for intermediate values of $n$. That the asymptotic series becomes not only unwieldy but unreliable in such cases is indicated by demonstrable errors in the results obtained by its use in one published paper. ${ }^{5}$ It is difficult to obtain accuracy from mechanical or numerical integration because of the oscillating integrand.

The author recently wanted numerical values of $P_{n}(r)$ for values of $n$ of the order of 10 . Since the accuracy of the asymptotic series and of mechanical or numerical integration was open to suspicion, a better method of evaluating the integral (1) was

${ }^{1}$ K. Pearson, Nature 72, 294 and 342 (1905).

${ }^{2}$ G. N. Watson, Theory of Bessel functions, Cambridge, Univ. Press, 1922, pp. 419-421.

${ }^{3}$ Nicholson, Quart. J. Pure Appl. Math., 48, 321-329 (1920).

${ }^{4}$ Phil. Mag. (6) 37, 321-347 (1919).

${ }^{5}$ G. D. Gillett, Some developments in common frequency broadcasting, Froc. I.R.E. 19, 1347-1369 (1931). Certain of the curves of Fig. 9 of this article fail to satisfy the easily proved relation $P_{n}(a)=1 /(n+1)$ when $a_{1}=a_{2}=\cdots=a_{n}=a$. 
sought. It was found possible to derive a convergent Fourier-Bessel series representation, which is simple in form and yields any accuracy desired. Derivation of the convergent series equivalent introduces some rather interesting analytical procedures and leads to a general method of evaluating certain types of Bessel integrals, particularly those which can be shown to vanish for all values of a parameter outside a certain finite range.

In the interests of simplicity we shall describe the method in terms of the special case of all vectors equal in length. The general case can be treated in a similar manner with more cumbersome details, of which only the final result will be stated here. We shall find it more convenient to calculate the probability $\Phi_{n}$ that the sum exceeds $r$. We set all the quantities $a_{m}(m=1,2, \cdots n)$ equal to each other and without loss of generality make them each equal to unity. Then

$$
\Phi_{n}(k)=1-P_{n}=1-k \int_{0}^{\infty} J_{1}(k t) J_{0}^{n}(t) d t=n \int_{0}^{\infty} J_{0}(k t) J_{1}(t) J_{0}^{n-1}(t) d t .
$$

The last form follows from an integration by parts. $\Phi_{n}(k)$ is the probability that the sum of $n$ vectors of equal lengths exceeds $k$ times the length of one vector. The equation (2) holds for all real non-negative values of $k$. It follows that $\Phi_{n}(k)$ vanishes for $k>n$ since the sum of $n$ equal vectors cannot be greater than $n$ times the length of one. The vanishing of $\Phi_{n}(k)$ for $k>n$ can also be shown directly from the infinite integral.

Let us multiply both sides of (2) by $k J_{0}(x k) d k$ and integrate with respect to $k$ from zero to infinity. We have the formal result:

$$
\int_{0}^{\infty} k J_{0}(x k) \Phi_{n}(k) d k=\int_{0}^{\infty} k J_{0}(x k) d k \int_{0}^{\infty} t J_{0}(k t) F(t) d t
$$

where

$$
F(t)=n J_{1}(t) J_{0}^{n-1}(t) / t .
$$

The integral on the left certainly exists and in fact satisfies sufficient conditions as a function of $x$ for the Fourier-Bessel integral identity to hold. ${ }^{6}$ It follows that:

$$
\int_{0}^{\infty} k J_{0}(x k) \Phi_{n}(k) d k=F(x)=\frac{n J_{1}(x)}{x} J_{0}^{n-1}(x) .
$$

We have thus found the Fourier-Bessel transform of $\Phi_{n}(k)$, or the Bessel analogue of the ordinary Fourier transform. We note that since $\Phi_{n}$ vanishes when $k$ exceeds $n$, we may also write:

$$
\int_{0}^{n} k J_{0}(x k) \Phi_{n}(k) d k=\frac{n J_{1}(x)}{x} J_{0}^{n-1}(x)
$$

or if we let $k=n z$,

$$
\int_{0}^{1} z J_{0}(n x z) \Phi_{n}(n z) d z=\frac{J_{1}(x)}{n x} J_{0}^{n-1}(x)
$$

${ }^{6}$ G. N. Watson, loc. cit., pp. 453-456. 
We now let $\Phi_{n}(n z)=f(z)$ and note that $f(z)$ in the interval $0 \leqq z \leqq 1$ satisfies a sufficient set of conditions ${ }^{7}$ for its representation as the sum of a convergent FourierBessel series:

$$
f(z)=\sum_{m=1}^{\infty} c_{m} J_{0}\left(j_{m} z\right), \quad 0 \leqq z \leqq 1
$$

where $j_{m}$ is the $m$ th root, in ascending order of magnitude, of the equation $J_{0}(x)=0$. The value of the coefficient $c_{m}$ is given by:

$$
c_{m}=\frac{2}{J_{1}^{2}\left(j_{m}\right)} \int_{0}^{1} z f(z) J_{0}\left(j_{m} z\right) d z=\frac{2 J_{1}\left(j_{m} / n\right) J_{0}^{n-1}\left(j_{m} / n\right)}{j_{m} J_{1}^{2}\left(j_{m}\right)} .
$$

The last form of the result is obtained by substituting the value $x=j_{m} / n$ in (7). Hence

$$
\Phi_{n}(k)=f(k / n)=2 \sum_{m=1}^{\infty} \frac{J_{1}\left(j_{m} / n\right) J_{0}^{n-1}\left(j_{m} / n\right)}{j_{m} J_{1}^{2}\left(j_{m}\right)} J_{0}\left(k j_{m} / n\right) .
$$

This is the result sought. It will be noted that $\Phi_{n}(n)=0$, as it should, since each term of the series then contains the factor $J_{0}\left(j_{m}\right)=0$. Incidentally, the vanishing of $\Phi_{n}(k)$ at $n=k$ is difficult if not impossible to show by asymptotic expansion. Values of $\Phi_{n}(k)$ computed from (10) are listed in Table I and plotted in Fig. 1.

\section{TABLE I}

\begin{tabular}{|c|c|c|c|c|c|c|}
\hline$k$ & $\Phi_{6}$ & $\Phi_{7}$ & $\Phi_{8}$ & $\boldsymbol{\Phi}_{\boldsymbol{x}}$ & $\Phi_{10}$ & $\Phi_{100}$ \\
\hline 1 & .8571 & .8750 & .8889 & .9000 & .9091 & \\
\hline 2 & .5335 & .5818 & .6211 & .6537 & .6812 & \\
\hline 3 & .2315 & .2866 & .3355 & .3788 & .4171 & \\
\hline 4 & .0636 & .0992 & .1356 & .1712 & .2055 & .8528 \\
\hline 5 & .0088 & .0515 & .0384 & .0581 & .0793 & \\
\hline 5.8 & .00013 & & & & & \\
\hline 6 & - & .0021 & .0067 & .0137 & .0229 & \\
\hline 6.6 & & .00015 & & & & \\
\hline 7 & & - & .00050 & .0020 & .0046 & .6138 \\
\hline 7.2 & & & .00025 & & & \\
\hline 7.5 & & & .00007 & & & \\
\hline 8 & & & & .00012 & .00056 & \\
\hline 9 & & & & - & .00005 & \\
\hline 10 & & & & & - & .3688 \\
\hline 15 & & & & & & .1052 \\
\hline 20 & & & & & & .01795 \\
\hline 25 & & & & & & .001802 \\
\hline 30 & & & & & & .000104 \\
\hline
\end{tabular}

$$
\text { Value of } \Phi_{n}(k a)=1-k \int_{0}^{\infty} J_{1}(k) J_{0}^{n}(z) d z=n \int_{0}^{\infty} J_{0}(k z) J_{1}(z) J^{n-1}(z) d z
$$

=probability that envelope of $n$ sine waves of equal amplitude exceeds $k$ times amplitude of one:

${ }^{7}$ G. N. Watson, loc. cit., pp. 591-592. 


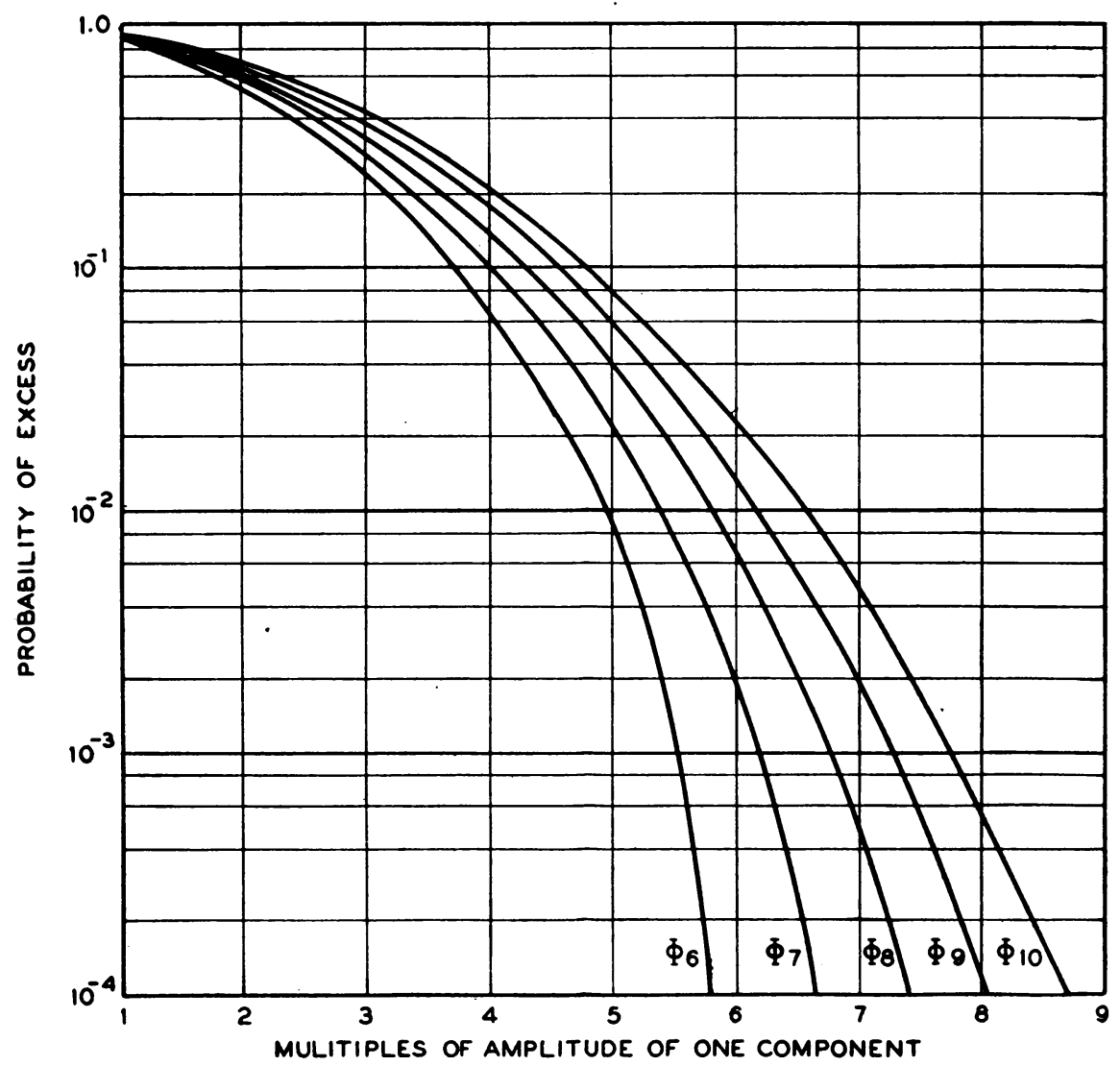

FIG. 1. $\Phi_{n}(k)=$ probability that the absolute value of the sum of $n$ vectors of equal magnitude and random phase exceeds $k$ times the absolute value of one component.

If the lengths of the vectors are unequal, the solution may be effected in a similar manner with the following results: Let

$$
A=\sum_{m=1}^{n} a_{m}
$$

where $a_{m}$ is the length of the $m$ th vector. Also let

$$
\begin{aligned}
b_{m} & =\sum_{s=1}^{n} J_{0}\left(a_{s} j_{m} / A\right), \\
c_{m} & =\sum_{s=1}^{n} \frac{a_{s} J_{1}\left(a_{s} j_{m} / A\right)}{J_{0}\left(a_{s} j_{m} / A\right)} .
\end{aligned}
$$

Then the probability that sum equals or exceeds $r$ is:

$$
\Phi_{n}=\frac{2}{A} \sum_{m=1}^{\infty} \frac{b_{m} c_{m} J_{0}\left(r j_{m} / A\right)}{j_{m} J^{2}\left(j_{m}\right)} .
$$

A general mathematical theorem of which the above results are special cases may be stated as follows: 
If

$$
\int_{0}^{\infty} J_{\nu}(r t) F(t) t d t=0 \text { for } \quad r>r_{0}
$$

and if $F(t)$ satisfies conditions enabling its representation by the Fourier-Bessel integral; then for $0<r<r_{0}$,

$$
\int_{0}^{\infty} J_{\nu}(r t) F(t) t d t=\frac{2}{r_{0}^{2}} \sum_{m=1}^{\infty} \frac{F\left(j_{m} / r_{0}\right) J_{\nu}\left(j_{m} r / r_{0}\right)}{J_{\nu+1}^{2}\left(j_{m}\right)}
$$

where

$$
J_{\nu}\left(j_{m}\right)=0, \quad j_{1}<j_{2}<j_{3}<\cdots .
$$

This completes the portion of our results dealing with the problem of random flights per se. It so happens that the primary object of the author is the application of the results to the envelope of a group of sine waves. As shown in the appendix the distribution function for the envelope of $n$ randomly phased sine waves of independent frequencies distributed over a range narrow compared with the frequency of any component is in fact the same $\Phi_{n}$ calculated above. It is of interest to compare the distribution of the envelope of a group of sine waves with the distribution of the instantaneous values. The latter distribution can be calculated by the method of characteristic functions. ${ }^{8}$ The probability density of the instantaneous value $E$ of the sine wave $a_{m} \cos \omega t$ is given by ${ }^{9}$

$$
p(E)=\left(a_{m}^{2}-E^{2}\right)^{-1 / 2} / \pi, \quad|E|<E_{0} .
$$

The "characteristic function" (or Fourier transform) of $p(E)$ is

$$
S_{m}(i \omega)=\frac{1}{2 \pi} \int_{-\infty}^{\infty} p(E) e^{-i \omega E} d E=\frac{J_{0}\left(a_{m} \omega\right)}{2 \pi} .
$$

For $n$ sine waves with peak amplitudes $a_{1}, a_{2}, \cdots, a_{n}$, the probability density is given by

$$
p_{n}(E)=(2 \pi)^{n-1} \int_{-\infty}^{\infty} e^{i E \omega} d \omega \prod_{m=1}^{n} S_{m}(i \omega)=\frac{1}{2 \pi} \int_{-\infty}^{\infty} e^{i E \omega} d \omega \prod_{m=1}^{n} J_{0}\left(a_{m} \omega\right) .
$$

This integral is not in convenient form to use the Fourier-Bessel integral and series. However, the presence of the exponential factor enables us to use the ordinary Fourier integral and series as follows: Multiply both sides of (20) by $e^{-i t E} d E$ and integrate from $-\infty$ to $\infty$. Then

$$
\int_{-\infty}^{\infty} p_{n}(E) e^{-i t E} d E=\frac{1}{2 \pi} \int_{-\infty}^{\infty} e^{-i t E} d E \int_{-\infty}^{\infty} e^{i E \omega} d \omega \prod_{m=1}^{n} J_{0}\left(a_{m} \omega\right)=\prod_{m=1}^{n} J_{0}\left(a_{m} t\right)
$$

by the Fourier integral identity. Since $p_{n}(E)=0$ for $|E|>A$,

$$
\int_{-A}^{A} p_{n}(E) e^{-i t E} d E=\prod_{m=1}^{n} J_{0}\left(a_{m} t\right) .
$$

${ }^{8}$ H. Cramér, Random variables and probability distributions, Cambridge Tract No. 36 (1937), Ch. IV.

${ }^{9}$ W. R. Bennett, J. Acoust. Soc. Am., 15, 164 (1944). 
Furthermore $p_{n}(E)$ satisfies a sufficient set of conditions for its expansion in a Fourier series $^{10}$ in the interval $-A<E<A$. Therefore:

$$
\begin{aligned}
p_{n}(E) & =\sum_{s=-\infty}^{\infty} c_{s} e^{i s \pi E / A}, \quad-A<E<A \\
c_{s} & =\frac{1}{2 A} \int_{-A}^{A} p_{n}(E) e^{-i s \pi E / A} d E=\frac{1}{2 A} \prod_{m=1}^{n} J_{0}\left(\frac{a_{m} s \pi}{A}\right) .
\end{aligned}
$$

The last step follows by substituting $t=s \pi / A$ in (22). Hence

$$
\begin{aligned}
p_{n}(E) & =\frac{1}{2 A} \sum_{s=-\infty}^{\infty} e^{i s \pi E / A} \prod_{m=1}^{n} J_{0}\left(s \pi a_{m} / A\right) \\
& =\frac{1}{2 A}\left[1+2 \prod_{s=1}^{\infty} \cos s \pi E / A \prod_{m=1}^{n} J_{0}\left(s \pi a_{m} / A\right)\right] .
\end{aligned}
$$

The probability $\Psi_{n}$ of finding an instantaneous absolute value exceeding $E$ is given by

$$
\begin{aligned}
\Psi_{n} & =1-2 \int_{0}^{E} p_{n}(E) d E \\
& =1-\frac{E}{A}-\frac{2}{\pi} \sum_{s=1}^{\infty} \frac{1}{s} \sin s \pi E / A \prod_{m=1}^{n} J_{0}\left(s \pi a_{m} / A\right) .
\end{aligned}
$$

If $a_{1}=a_{2}=\cdots=a_{n}=a, \Psi_{n}(k)$ is the probability of exceeding $k a$,

$$
\begin{aligned}
\Psi_{n}(k) & =1-\frac{2}{\pi} \int_{0}^{\infty} J_{0}^{n}(z) \frac{\sin k z}{z} d z \\
& =1-\frac{k}{n}-\frac{2}{\pi} \sum_{s=1}^{\infty} \frac{1}{s} J_{0}^{n}(s \pi / n) \sin s \pi k / n .
\end{aligned}
$$

Values of $\Psi_{n}(k)$ are listed in Table II.

\section{TABLE II}

$$
\text { Value of } \Psi_{n}(k)=1-\frac{2}{\pi} \int_{0}^{\infty} J_{0}^{n}(z) \frac{\sin k}{z} d z
$$
of one.

$=$ probability that instantaneous absolute value of $n$ sine waves of equal peaks exceeds $k$ times peak

\begin{tabular}{c||c|c|c|c|c|c|c|c}
\hline$k$ & 1 & 2 & 3 & 4 & 5 & 6 & 7 & 8 \\
\hline$\Psi_{10}$ & .6604 & .3779 & .1832 & .07330 & .02337 & .00563 & .00094 & .00009 \\
\hline
\end{tabular}

${ }^{10}$ This method of evaluating integrals by Fourier series has also been used by D. G. Kendall, $A$ summation formula associated with finite trigonometric integrals, Quart. J. Math. (Ox. Ser.) 13, 172-184 (1942). 
It is convenient to express results in terms of the ratio of the quantity to the root mean square value. Let $\eta$ represent the root mean square value of the envelope and $\tau$ the root mean square instantaneous value of $n$ sine waves with equal amplitude $a$. Then

$$
\begin{aligned}
& \eta=a \sqrt{n} \\
& \tau=a \sqrt{n / 2}
\end{aligned}
$$

Fig. 2 shows $\Phi_{n}(k)$ plotted against $10 \log _{10} k^{2} / n$, the ratio of $k a$ to $\eta$ expressed in

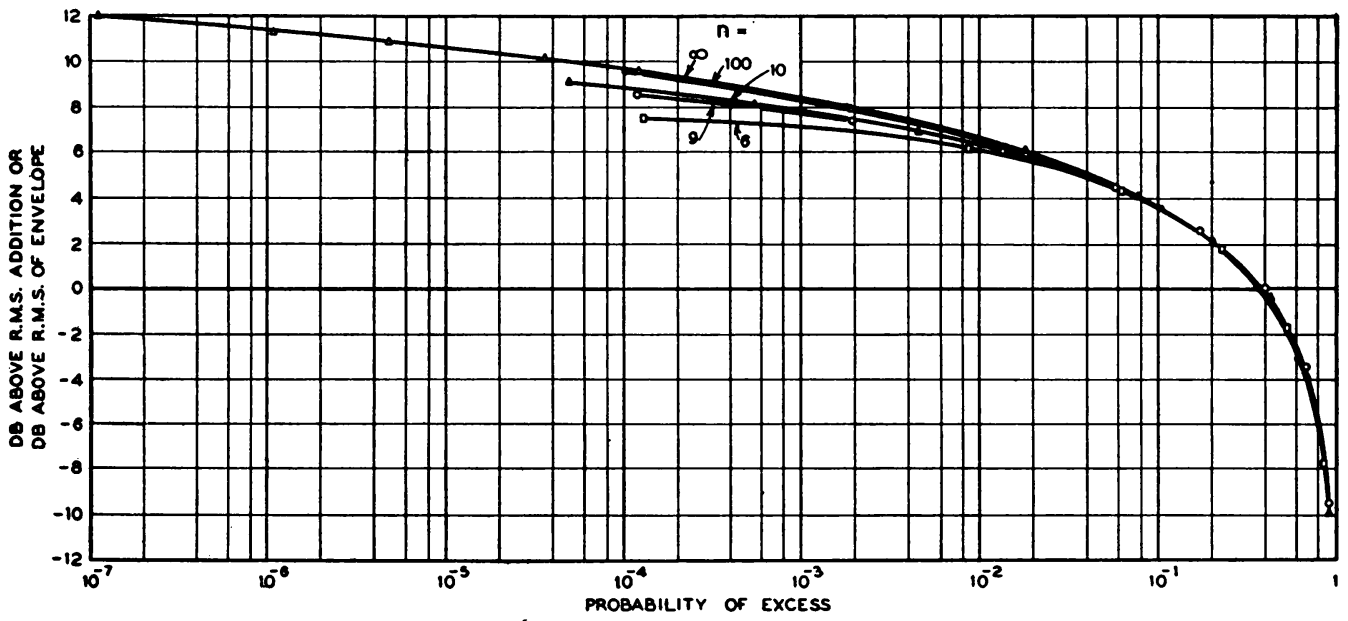

Fig. 2. Distribution function of envelope of $n$ equiamplitude randomly phase sine waves expressed in $\mathrm{db}$ above root mean square of envelope.

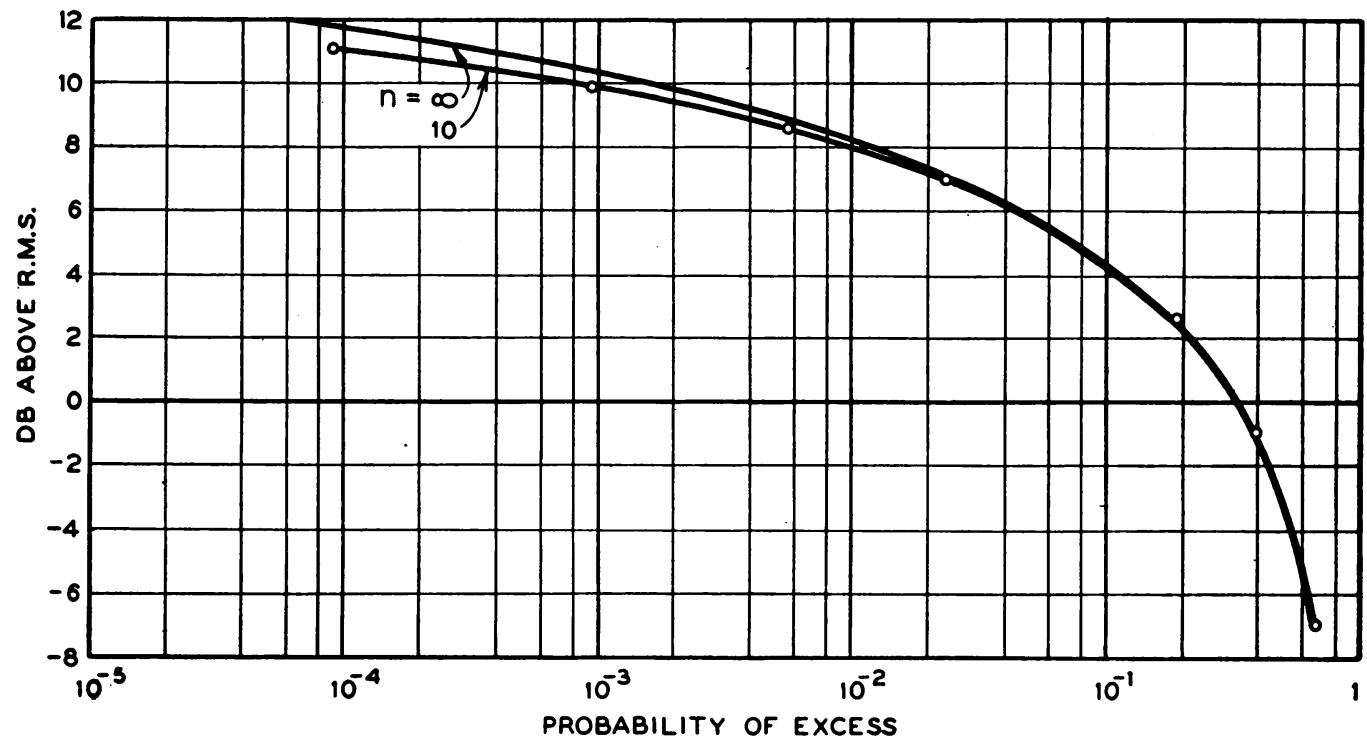

Fig. 3. Distribution function of instantaneous value of $n$ equiamplitude randomly phased sine waves expressed in $\mathrm{db}$ above root mean square. 
decibels. Likewise Fig. 3 shows $\Psi_{n}(k)$ plotted against $10 \log _{10} 2 k^{2} / n$, the ratio of $k a$ to $\tau$ expressed in decibels. The limiting forms of these curves when $n$ is large ${ }^{11}$ are:

$$
\begin{aligned}
& \lim _{n \rightarrow \infty} \Phi_{n}(k)=e^{-k^{2} / n} \\
& \lim _{n \rightarrow \infty} \Psi_{n}(k)=1-\operatorname{erf} k / \sqrt{n} .
\end{aligned}
$$

The latter approaches the normal law in one dimension, while the former approaches the distribution of the radial coordinate in a two-dimensional normal field. The results are in accordance with the central limit theorem. ${ }^{12}$ All numerical calculations of this paper were made by Mrs. A. J. Shanklin.

Since completion of this work, a published article $^{13}$ has appeared treating the same problems by different methods. Figs. 4 and 5 of the reference are graphs of the function we have called $\Psi_{n}$ and Figs. 7 and 8 give $\Phi_{n}$. Agreement between these curves and Tables I and II of the present paper is good except near the maximum amplitudes, where the probabilities plotted on the curves are higher than our tabulated values. The latter have been carefully checked and are believed to be accurate to the number of significant figures listed. Upper bounds have been calculated for the remainder after the actual number of terms used in the series and shown to be negligibly small.

\section{APPENDIX}

\section{Envelope of a Group of Sine Waves}

Let

$$
E=\sum_{m} E_{m} \cos p_{m} t
$$

Then by obvious reariangements:

$$
\begin{aligned}
E= & \operatorname{Re} \sum_{m} E_{m} e^{i p_{m} t}=\operatorname{Re} e^{i p_{0} t} \sum_{m} e^{i\left(p_{m}-p_{0}\right) t} \\
= & \cos p_{0} t \sum_{m} E_{m} \cos \left(p_{m}-p_{0}\right) t-\sin p_{0} t \sum_{m} E_{m} \sin \left(p_{m}-p_{0}\right) t \\
= & \left\{\left[\sum_{m} E_{m} \cos \left(p_{m}-p_{0}\right) t\right]^{2}+\left[\sum_{m} E_{m} \sin \left(p_{m}-p_{0}\right) t\right]^{2}\right\}^{1 / 2} \\
& \cdot \cos \left[p_{0} t+\arctan \frac{\sum_{m} E_{m} \sin \left(p_{m}-p_{0}\right) t}{\sum_{m} E_{m} \cos \left(p_{m}-p_{n}\right) t}\right] .
\end{aligned}
$$

This is the familiar form of an envelope function multiplying an oscillating function. In order for the resolution to be physically significant, $p_{0}$ must be large compared

${ }^{11}$ The limiting forms can be derived from the integrals by Laplace's process as described in Watson, loc. cit., Sec. 13.48 .

${ }^{12} \mathrm{H}$. Cramér, loc. cit. Chap. X, J. V. Uspensky, Introduction to mathematical probability, McGrawHill, New York, Chap. 15.

${ }^{13}$ Margaret Slack, The probability distributions of sinusoidal oscillations combined in random phase, J.I.E.E., 93, 76-86 (1946). 
with $p_{m}-p_{0}$. The same expression for the envelope function may be derived from the mathematical definition of an envelope if we regard the defining equation for $E$ as that of a family of curves in $E$ and $t$ with the parameter $\left(p_{m}-p_{0}\right)=\alpha$. Eliminating $\alpha$ between this equation and the equation obtained by differentiating partially with respect to $\alpha$ gives the square root function appearing above.

The envelope is seen to be the square root of the sum of the squares of the sum of a set of horizontal components $E_{m} \cos \left(p_{m}-p_{0}\right) t$ and the sum of a set of vertical components $E_{m} \sin \left(p_{m}-p_{0}\right) t$. It is therefore equal to the magnitude of the resultant of a set of two-dimensional vectors with absolute values $E_{m}$ and phase angles, $\left(p_{m}-p_{0}\right) t$. If the frequencies $p_{1}, p_{2}, \cdots$ are incommensurable, the phases are random and the problem is the same as that of random flights.

We note that the square of the envelope is equal to

$$
\sum_{m} E_{m}^{2}+2 \sum_{l, m}^{\prime} E_{l} E_{m} \cos \left(p_{l}-p_{m}\right) t
$$

where $\sum^{\prime}$ does not include $l=m$. The average square is therefore $\sum_{m} E_{m}{ }^{2}$. 